\title{
High glucose upregulates myosin light chain kinase to induce microfilament cytoskeleton rearrangement in hippocampal neurons
}

\author{
LIYING ZHU ${ }^{1,2 *}$, CHENGCHENG LI $^{1 *}$, GUIQIN DU ${ }^{1}$, MEIXIU PAN ${ }^{1}$, GUOQI LIU ${ }^{1}$, WEI PAN ${ }^{1,2}$ and XING LI ${ }^{1,2}$ \\ ${ }^{1}$ Department of Medical Laboratory, Guizhou Medical University; ${ }^{2}$ Department of Medical Laboratory, \\ Affiliated Hospital of Guizhou Medical University, Guiyang, Guizhou 550004, P.R. China
}

Received October 20, 2017; Accepted February 19, 2018

DOI: $10.3892 / \mathrm{mmr} .2018 .8960$

\begin{abstract}
Chronic hyperglycemia leads to myosin light chain kinase (MLCK) upregulation and induces neuronal damage. However, the underlying molecular mechanism of neuronal damage in hyperglycemia has not yet been fully elucidated. In the present study, hippocampal neuronal cells were cultured and treated with a high glucose concentration $(45 \mathrm{mmol} / \mathrm{l})$. The results demonstrated that high glucose induced shrinking of the synapses, nuclear shape irregularity and microfilament damage. Filamentous actin (F-actin) filaments were rearranged, cell apoptosis rate was increased and the protein expression of MLCK and phosphorylated (p)-MLC was upregulated. The MLCK inhibitor ML-7 largely reversed the alterations in the microfilament cytoskeleton, inhibited F-actin depolymerization, reduced apoptosis and downregulated MLCK and p-MLC protein expression. Overall, these results indicated that high glucose upregulated MLCK to promote F-actin depolymerization, which induced microfilament cytoskeleton rearrangement in hippocampal neuronal cells.
\end{abstract}

\section{Introduction}

Diabetic encephalopathy (DE) is one of the most prevalent chronic complications of diabetes mellitus (DM) and is characterized by degeneration and dysfunction of the central nervous system. Numerous studies have reported that chronic hyperglycemia not only leads to inhibition of glucose metabolism in the brain (1), however also results in neuronal damage, leading to

Correspondence to: Professor Wei Pan or Professor Xing Li, Department of Medical Laboratory, Guizhou Medical University, 9 Beijing Road, Guiyang, Guizhou 550004, P.R. China

E-mail:pw_6602@sina.com

E-mail: lixingsci@sina.com

*Contributed equally

Key words: cell primary culture, hippocampal neurons, myosin light chain kinase, microfilament cytoskeleton, filamentous actin cognitive function impairment in rats (2). Diabetes-associated cognitive decline is a central nervous system complication induced by DM (3). DE increases the probability of cognitive decline, as well as acceleration of Alzheimer's disease and other forms of dementia $(4,5)$.

The neuronal cytoskeleton is a fibrous protein filament structure responsible for information transmission, cell shape maintenance, energy conversion, cell movement and other cellular functions. The neuronal cytoskeleton is primarily composed of microtubules (MT), microfilaments (MF) and neurofilaments. The primary component of MF is filamentous actin (F-actin). F-actin polymerization and depolymerization take place during cytoskeletal remodeling and may to a certain extent, reflect the functional status of cells $(6,7)$. Previous studies identified certain tau species to be associated with compromised MT integrity and impaired neuronal function, leading to long-lasting cellular degradation and cognitive deficits (8-10). McLean et al (11) revealed a number of post-translational modifications of neuronal cytoskeletal proteins that may contribute to the altered axonal transport and subsequent nerve dysfunction in experimental diabetes. Phosphorylation of microtubule-associated protein (MAP) 2 induced by glycogen synthase kinase 3 modulates its association with MT and regulates MT stability (12). Chen et al (13) observed that mice with DM exhibited a decrease in MAP 2 protein expression in the hippocampus and cerebral cortex. Advanced glycation end-products (AGEs)/AGEs receptor promote MT stabilization via the suppression of the NAD-dependent protein deacetylase sirtuin-2/acetylated $\alpha$-tubulin signaling pathway during development of diabetic cardiomyopathy (14).

The authors previously demonstrated that myosin light chain kinase (MLCK) is upregulated in the brain tissues and vascular walls of diabetic rats using two-dimensional electrophoresis and mass spectrometry detection technology (7). The results demonstrated a significant positive association between MLCK and development of DM (7). These results also indicate that MLCK may be associated with the occurrence and development of cognitive dysfunction in diabetes (7). As a serine/threonine kinase, MLCK modulates the phosphorylation of myosin light chain (p-MLC), induces rearrangement of the hippocampal neuronal cytoskeleton and is involved in cell 
movement, migration and apoptosis (15). MLCK may affect material transport, energy conversion, information transfer and cell differentiation of vascular smooth muscle cells (16-18).

In the present study, the primary hippocampal neurons were cultured in a high-glucose environment and the alterations in cellular morphology and microfilament cytoskeleton were evaluated. Furthermore, MLCK and p-MLC protein expression levels were investigated to determine how MLCK affects the hippocampal neuronal microfilament structure in a high-glucose environment, with the aim to elucidate the pathogenesis of diabetic cognitive dysfunction and offer a novel approach to clinical treatment.

\section{Materials and methods}

Experimental animals. A total of 100 healthy newborn (24h-old) Sprague-Dawley rats (5-10 g) were purchased from the Experimental Animal Center of Guizhou Medical University (Guiyang, China). Prior to the experiments, the animals were acclimatized in a temperature- and humidity-controlled environment with food and water (ad libitum) under a $12 \mathrm{~h}$ light/dark cycle. Of the 100 rats, 44 were female and 56 were male. All experiments were approved by the Ethics Committee of Guizhou Medical University (approval no. 1702093).

Hippocampal neuronal cell preparation and culture. Hippocampal neurons were isolated from newborn SD rats and digested with $0.125 \%$ trypsin, as previously described (19). The hippocampal neurons were subsequently cultured in basal cell culture medium $[0.5 \mathrm{ml}$ penicillin-streptomycin solution, $0.5 \mathrm{ml}$ L-glutamine, $2.5 \mathrm{ml}$ fetal bovine serum (Gibco; Thermo Fisher Scientific, Inc., Waltham, MA, USA), $5 \mathrm{ml}$ horse serum (Gibco; Thermo Fisher Scientific, Inc.) and $41.5 \mathrm{ml}$ Dulbecco's modified Eagle medium (Gibco; Thermo Fisher Scientific, Inc.)]. The cells were incubated at $37^{\circ} \mathrm{C}$ with $5 \% \mathrm{CO}_{2}$. After $6 \mathrm{~h}$ the medium was changed to maintenance culture medium [0.5 ml penicillin-streptomycin solution, $0.5 \mathrm{ml} \mathrm{L}$-glutamine, $1 \mathrm{ml} \mathrm{B} 27$ and $48 \mathrm{ml}$ Neurobasal-A culture medium (Gibco; Thermo Fisher Scientific, Inc.)]. Every 2 days, half of the culture medium was replaced with fresh medium. The cells were incubated at $37^{\circ} \mathrm{C}$ with $5 \% \mathrm{CO}_{2}$.

Identification of hippocampal neurons. Hippocampal neuronal cells were cultured using the aforementioned procedure. After 7 days, the cells were rinsed three times with PBS, fixed for 30 min with $4 \%$ paraformaldehyde at room temperature, incubated for 5-10 min with $3 \% \mathrm{H}_{2} \mathrm{O}_{2}$ deionized water at room temperature and immuno-stained with anti-neuron-specific enolase (NSE) antibody (1:100; M02930; Wuhan Boster Biological Technology, Ltd., Wuhan, China) at room temperature, followed by the addition of goat anti-rabbit immunoglobulin G (IgG) secondary antibody (1:200, BA1001; Wuhan Boster Biological Technology, Ltd.) and visualized using fluorescence microscopy (magnification, x400). ImageJ $1.45 \mathrm{~s}$ (National Institutes of Health, USA) was used to estimate the purity of neurons.

Transmission electron microscopy (TEM) of hippocampal neuronal cells. The primary cultured hippocampal neurons were divided into the control (untreated), high-glucose
(45 mmol/1), and high-glucose+MLCK inhibitor (ML-7; $10 \mathrm{mmol} / 1$ Sigma-Aldrich, Merck KGaA, Darmstadt, Germany) groups. A previous study evaluated the effects of MLCK under conditions of differing glucose concentrations (20). The preset study reported that the exposure of hippocampal neurons to $45 \mathrm{mmol} / \mathrm{l}$ glucose did not affect cell viability, however increased the expression of MLCK; therefore, $45 \mathrm{mmol} / \mathrm{l}$ glucose was selected as the high-glucose group (data not shown).

After 5 days, $45 \mathrm{mmol} / 1$ glucose was added to the high-glucose group, and $45 \mathrm{mmol} / 1$ glucose with $10 \mathrm{mmol} / \mathrm{l}$ ML-7 was added to the high-glucose + ML-7 group. After 2 days, differentially treated hippocampal neuronal cells were fixed with $2.5 \%$ glutaraldehyde in $0.2 \mathrm{M}$ cacodylate buffer (pH 7.0) for $2 \mathrm{~h}$ at $4^{\circ} \mathrm{C}$, and subsequently washed in a solution of $0.2 \mathrm{M}$ cacodylate buffer, sucrose and distilled water overnight at $4^{\circ} \mathrm{C}$. The samples were postfixed in $1 \%$ osmium tetroxide in $0.2 \mathrm{M}$ cacodylate buffer for $2 \mathrm{~h}$ at $4^{\circ} \mathrm{C}$, washed with distilled water and stained with $2 \%$ aqueous uranyl acetate for $1 \mathrm{~h}$ at $4^{\circ} \mathrm{C}$. Subsequently, the samples were washed with distilled water and stained with $2 \%$ aqueous uranyl acetate for $20 \mathrm{~min}$ at room temperature, dehydrated through a graded ethanol series (30, 50, 70, 80,96 and 99\%; 15 min each), then incubated twice with propylene oxide for $15 \mathrm{~min}$ at room temperature. Finally, the cell pellets were embedded in epoxy resin for $24 \mathrm{~h}$ at $60^{\circ} \mathrm{C}$, and ultra-thin sections $(70 \mathrm{~nm})$ were cut by a Leica UC6 ultramicrotome (Leica Microsystems GmbH, Wetzlar, Germany) and transferred to copper grids (200 mesh). Following staining with uranyl acetate for $5 \mathrm{~min}$ and lead citrate for $1 \mathrm{~min}$ at room temperature, the sections were examined with a transmission electron microscope (JEOL, Ltd., Tokyo, Japan; $80 \mathrm{kV}$ ) and the images were captured with a digital camera (BioScan model 792; Gatan, Inc., Pleasanton, CA, USA).

Cell apoptosis analysis. Differentially treated hippocampal neuronal cells were cultured in serum-free medium (Gibco; Thermo Fisher Scientific, Inc.) for 48 h. Subsequently, apoptosis was assessed with the Annexin V-7AAD apoptosis detection kit (Nanjing KeyGen Biotech Co., Ltd., Nanjing, China) according to the manufacturer's protocol. Cells were analyzed using fluorescence-activated cell sorting cytometry (BD Biosciences, Franklin Lakes, NJ, USA). GraphPad Prism 5 (GraphpPad Software, Inc., La Jolla, CA, USA) was used to analyze the results.

Immunofluorescence study. Glass slides (14x14-mm) were placed into 24-well plates (Corning Life Sciences, Corning, NY, USA). Differentially treated hippocampal neuronal cells were cultured on $14 \times 14-\mathrm{mm}$ glass slides for $48 \mathrm{~h}$ using the aforementioned procedure. After $48 \mathrm{~h}$, the cells were fixed on 14x14-mm glass slides with $4 \%$ paraformaldehyde for $30 \mathrm{~min}$ at room temperature then washed with PBS. Following blocking with 3\% goat serum (Wuhan Boster Biological Technology, Ltd.) for $30 \mathrm{~min}$ at room temperature. The cells were incubated with antibodies specific for F-actin (1:100; ab205; Abcam, Cambridge, UK) overnight at $4^{\circ} \mathrm{C}$. Following three washes with PBS, the cells were incubated with goat anti-mouse IgG antibodies (Alexa Fluor488; cat. no. 4408S, Cell Signaling Technology, Inc., Danvers, MA, USA) for $1 \mathrm{~h}$ at $37^{\circ} \mathrm{C}$ and then the slides were mounted by adding DAPI-Fluoromount-G 

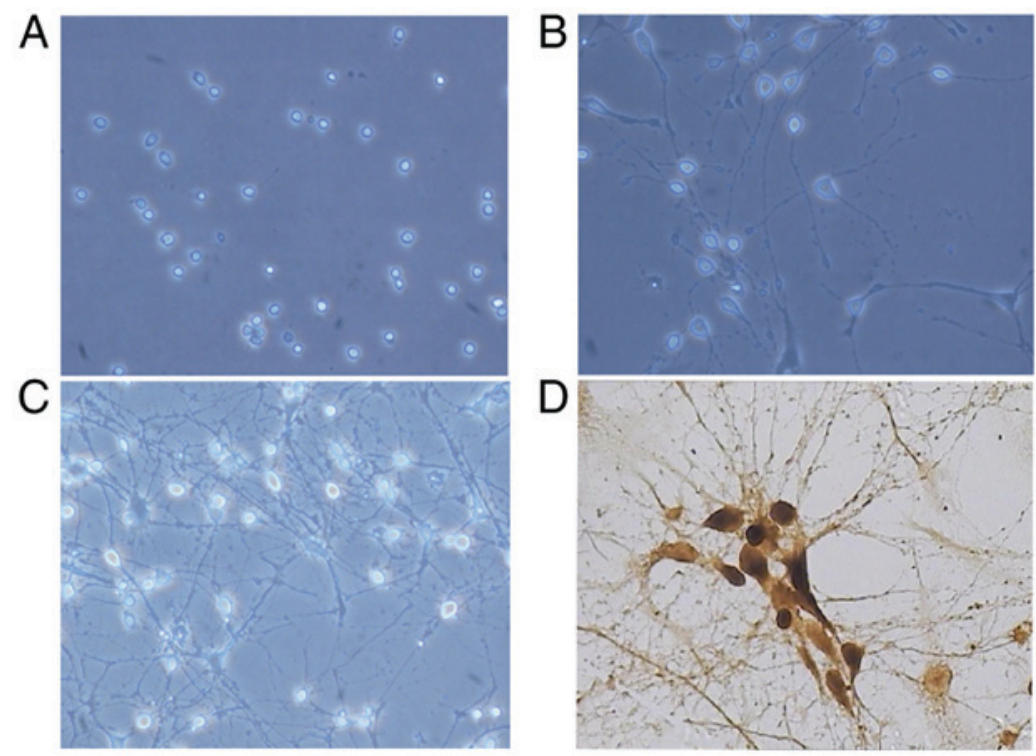

Figure 1. Morphology and identification of hippocampal neurons at different time points. (A) Cells exhibited a round, oval or tapered shape, and the cell bodies appeared bright and full, three-dimensional and with strong refraction; day 1 , magnification x200. (B) Volume of the cells was visibly increased, and the neurites are branched; day 4, magnification x200. (C) Neurites were branched and overlapping; day 7, magnification x200. (D) Hippocampal neuronal cells were identified following immunocytochemical staining with anti-neuron-specific enolase; magnification x400.

(1:1,000; Wuhan Sanying Biotechnology, Inc., Wuhan, China) for $5 \mathrm{~min}$ at room temperature. Subsequently, cells were examined under a confocal laser scanning microscope (magnification, x630). ImageJ software 1.45s (National Institutes of Health) was used to analyze the results.

Western blot analysis. Total proteins were extracted from differentially treated hippocampal neuronal cells using radioimmunoprecipitation assay buffer (Beyotime Institute of Biotechnology) on ice. The protein concentration was determined with BCA Protein Assay kit (Beyotime Institute of Biotechnology). The proteins $(50 \mu \mathrm{g})$ were isolated by $12 \%$ SDS-PAGE at room temperature. Following electrophoresis, the proteins were transferred onto polyvinylidene membranes (Thermo Fisher Scientific, Inc.) for $1.5 \mathrm{~h}$. The membranes were incubated with 5\% non-fat milk in TBS for $1.5 \mathrm{~h}$ at room temperature, followed by incubation with primary antibodies against MLCK (1:5,000; ab76092; Abcam), p-MLC (1:1,000; cat. no. 3675; Cell Signaling Technology, Inc.) and $\beta$-actin (1:2,000; BS6007MH; Biogot Technology Co., Ltd., Nanjing, China) overnight at $4^{\circ} \mathrm{C}$. Subsequently, the membranes were incubated with horseradish peroxidase-conjugated goat anti-mouse IgG secondary antibody $(1: 2,000$; Bioworld Technology, China) for $2 \mathrm{~h}$ at room temperature. Bands were visualized with enhanced chemiluminescence western blot detection reagents (Advansta, Inc., Menlo Park, CA, USA) and analyzed using the Image-Pro Plus V6.0 software (Media Cybernetics, Inc., Rockville, MD, USA).

Statistical analysis. Statistical analyses were performed using SPSS software, version 17.0 (SPSS, Inc., Chicago, IL, USA). Data were analyzed using one-way analysis of variance with the Bonferroni post hoc test. Each experiment was performed three times and the results are presented as the mean \pm standard deviation. $\mathrm{P}<0.05$ was considered to indicate a statistically significant difference.

\section{Results}

Morphology and identification of hippocampal neurons. The hippocampal neuronal cells were successfully isolated from newborn SD rats and grown in culture. After 6 h, $90 \%$ of the cells had attached to the dish. The cells exhibited a round, oval or tapered shape, and the cell bodies were clearly visible and had a three-dimensional appearance with strong refraction (Fig. 1A). After 4 days, the volume of the cells had distinctly increased and the neurites were branched (Fig. 1B). After 7 days, the neurites were branched and appeared to be overlapping (Fig. 1C). The purity of hippocampal neurons was measured by immunohistochemical staining with anti-NSE antibody and was determined to be $>90 \%$ (Fig. 1D).

Ultrastructure of hippocampal neurons. To determine whether the ultrastructure of hippocampal neurons was altered when they were cultured in a high-glucose environment, the neurons were examined using TEM. In the control group, the nuclear morphology was regular and the chromatins in the nucleus were abundant and homogeneously distributed. The cell morphology was normal and the cell organelles were abundant. The endoplasmic reticulum and mitochondria were normal in morphology, and there were large number of microfilaments in the cytoplasm (Fig. 2A). In the high-glucose group, the nuclear membranes were shrunk, the morphology of the nucleus was irregular, and the chromatins were heterogeneously distributed, granular and aggregated into blocks. The cytoplasm was fragmented, the endoplasmic reticulum appeared edematous and the mitochondria were markedly enlarged. These alterations were generally accompanied by dilatation of the Golgi complex and were frequently accompanied by autophagy and appearance of dense bodies (Fig. 2B). In the high-glucose + ML-7 group, the cell morphology was almost normal. The nucleus was normal, with a round or oval shape and the chromatins were abundant. The endoplasmic reticulum and Golgi complex were 
A

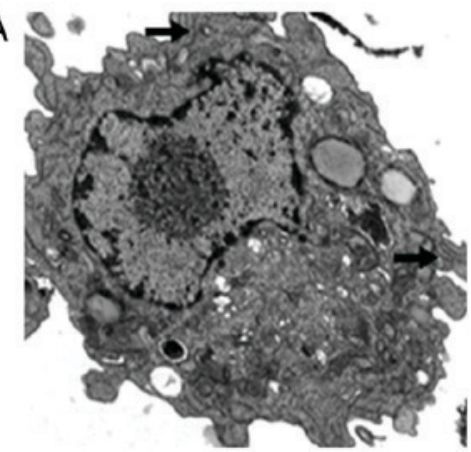

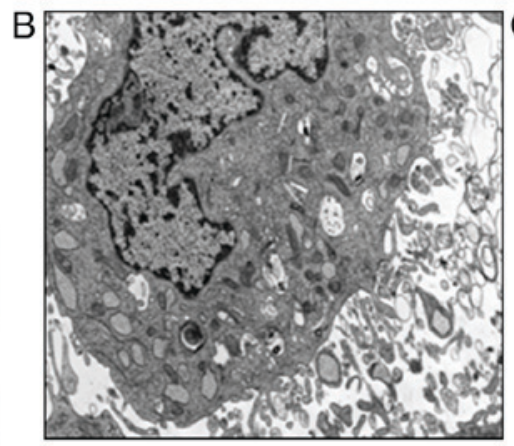

Figure 2. Ultrastructure of hippocampal neurons in culture. (A) In the control group, the morphology of the nucleus was regular, and the chromatins in the nucleus were abundant and homogeneously distributed. The cell organelles were abundant, the endoplasmic reticulum and mitochondria were normal in morphology, and there were large numbers of microfilaments in the cytoplasm (magnification, x10,000). Black arrow, microfilament structural integrity. (B) In the high-glucose group, a large fragment of a relatively well-preserved perikaryon of the pyramidal neuron was observed. Certain mitochondria appeared to be enlarged. The nuclear membrane appeared to have shrunk (magnification, x10,000). (C) In the high glucose+ML-7 group the nuclei were normal, round or oval, with abundant chromatin. The endoplasmic reticulum and Golgi complex were only partly edematous. The mitochondria were abundant and their morphology was normal (magnification, x10,000). Red circles, abundant microfilament.
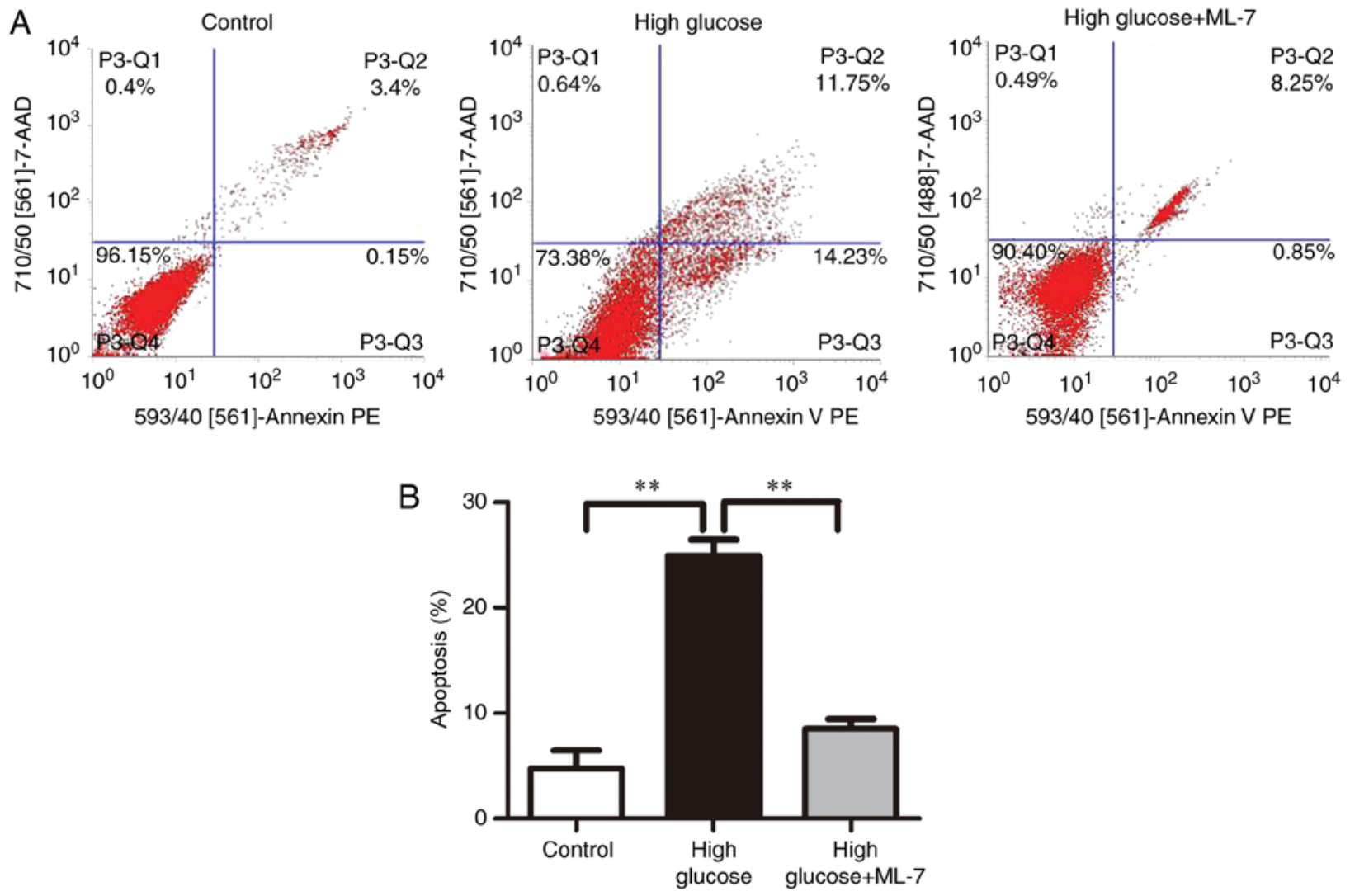

Figure 3. Apoptosis rates in different groups. (A) Representative images of flow cytometry analysis of hippocampal neurons in different groups. (B) The bar graph presents the percentage of apoptotic cells. The experiments were performed in triplicate; the data are expressed as the mean \pm standard deviation. ${ }^{* * *} \mathrm{P}<0.01$.

only partly edematous. The mitochondria were abundant and their morphology was normal. The cytoplasmic volume of these neurons was increased. Compared with the high-glucose group, the damaged structural elements had mostly recovered in the high glucose + ML-7 group (Fig. 2C).

Apoptosis rate in different groups. In order to determine the cause of cell death following the ultrastructural alterations of the hippocampal neurons, flow cytometry was performed to examine cell apoptosis among different groups. The results revealed that the apoptosis rates were $4.75 \pm 1.20$, $24.91 \pm 1.07$ and $8.5 \pm 0.63 \%$ in the control, high-glucose and high glucose + ML-7 groups, respectively (Fig. 3A and B), suggesting that high glucose induced hippocampal neuron apoptosis.

F-actin cytoskeleton organization. In the control group, F-actin was distributed homogeneously, surrounding the cell body and dendritic spines and forming a complete and continuous actin band (Fig. 4A). In the high-glucose group, the actin bands had 


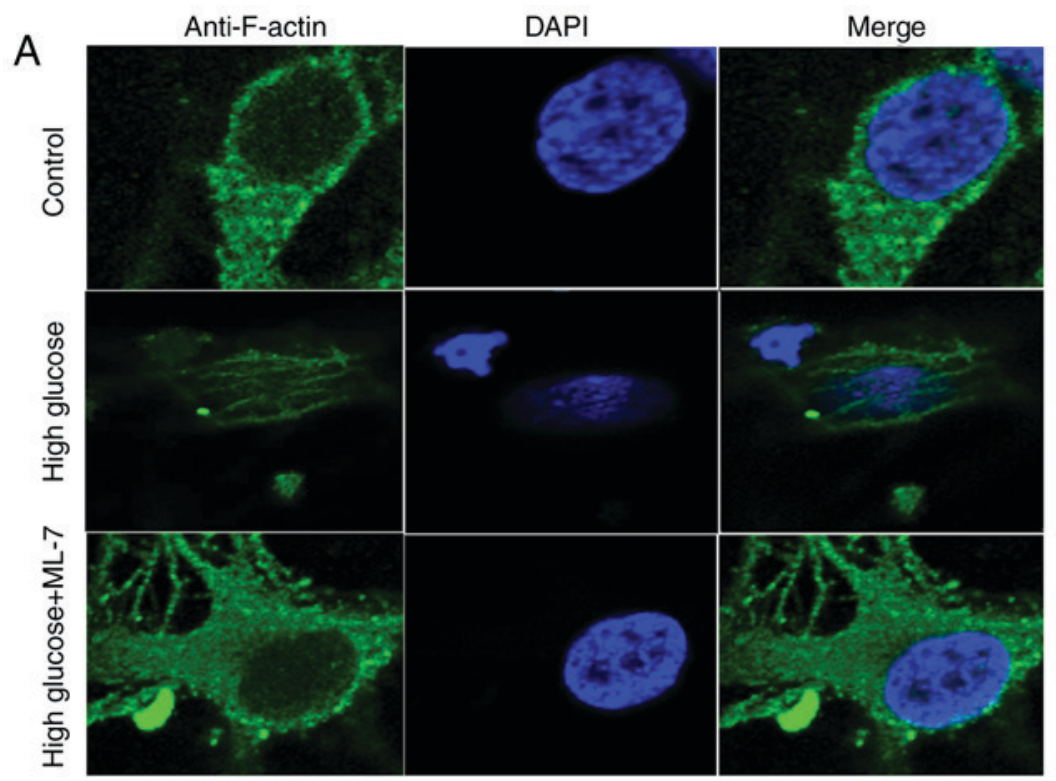

B

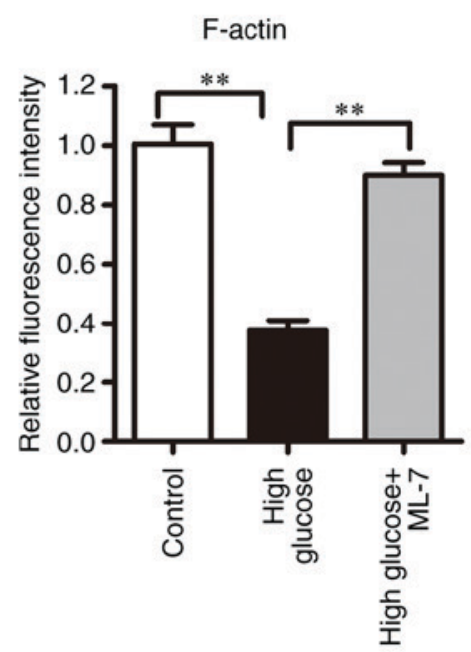

Figure 4. Structural alterations of F-actin in hippocampal neurons. (A) Representative images of immunofluorescent staining for F-actin (green) with DAPI nuclear staining (blue) in various groups (magnification, $\mathrm{x} 630$ ). (B) Quantitative analysis of fluorescence intensity of F-actin. ${ }^{* *} \mathrm{P}<0.01$. F-actin, filamentous actin.
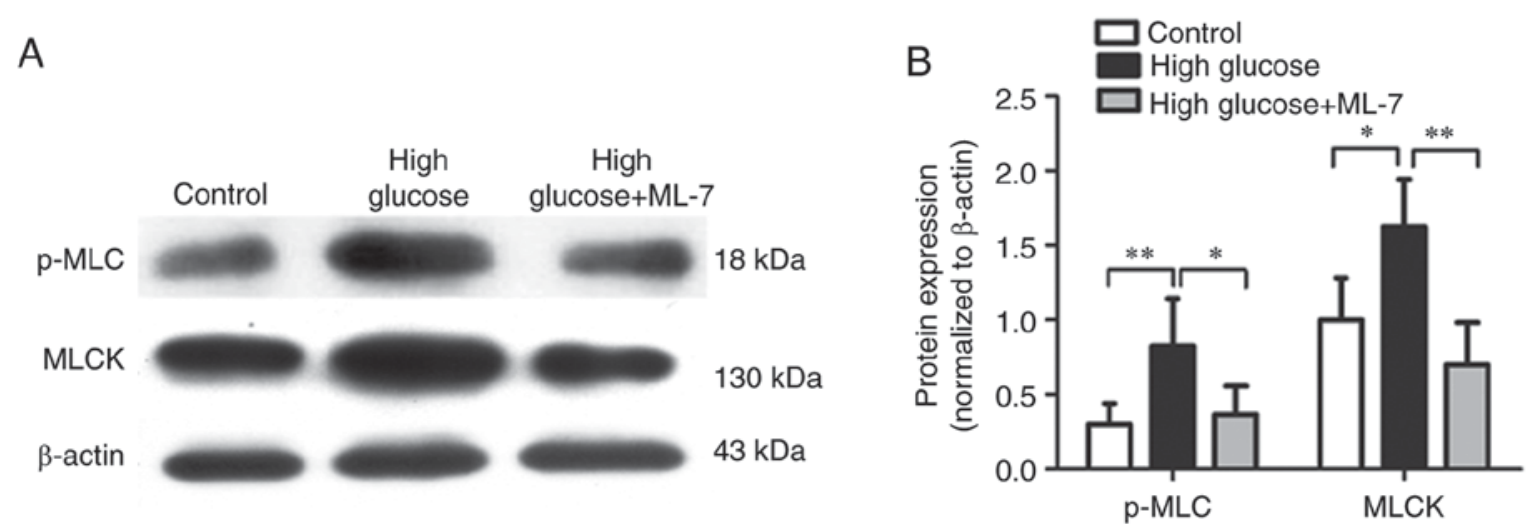

Figure 5. MLCK and p-MLC protein expression in various groups. (A) Western blot analysis of MLCK and p-MLC protein expression in various groups, using $\beta$-actin as an endogenous control. (B) Quantitative analysis of MLCK and p-MLC protein expression levels; ${ }^{*} \mathrm{P}<0.05$ and ${ }^{* *} \mathrm{P}<0.01$. MLCK, myosin light chain kinase; p-MLC, phosphorylated myosin light chain.

disappeared and the F-actin was depolymerized or rearranged. In addition, the dendrites and axons were shrunk. Compared with the high-glucose group, in the high-glucose + ML-7 group the F-actin bands were recovered and F-actin was apparent in dendritic spines, although it was abnormally arranged. To calculate the F-actin density in different groups, the fluorescence intensity was calculated in the cell area excluding the nucleus. Compared with the control group, the density of F-actin was lower in the high-glucose group (from three cell preparations; $\mathrm{P}<0.01)$. Compared with the high-glucose group, the density of F-actin increased in the high-glucose + ML-7 group $(\mathrm{P}<0.01$; Fig. 4B). The aforementioned results indicated that ML-7 may induce F-actin polymerization.

Protein expression of $M L C K$ and $p-M L C$ in hippocampal neurons. To investigate whether high glucose affects MLCK, the protein expression of MLCK and p-MLC were measured by western blot analysis. Compared with the control group, the protein expression of MLCK and p-MLC were upregulated in the high-glucose group. By contrast, compared with the high-glucose group, the expression of MLCK and p-MLC were downregulated in the high-glucose + ML-7 group (Fig. 5A and B).

\section{Discussion}

DE is characterized by degeneration and dysfunction of the central nervous system. Neuronal plasticity serves a role in the brain and refers to morphological, biochemical and physiological alterations in the developing nervous system. Accumulating evidence indicates that alterations in synaptic contacts modulate the function of the nervous system $(21,22)$.

In recent years, studies of the cytoplasm have focused on the organizational properties and functional significance of actin-containing microfilaments. Actin is the primary component of microfilaments, whereas actin and myosin molecules are the main components of non-muscle cells (23-25). In non-muscle cells, actin is present in the form 
of polymerized filaments (F-actin) and in the globular form of G-actin (23). In order to be physiologically active, actin must be polymerized in filaments (23). The viscosity of the cytoplasm is determined by the state of actin polymerization (23). The presence or absence of myosin molecules determines whether actin filaments will be involved in movement generation (23). Myosin has been identified in neurons and non-muscle cells in biochemical experiments. The contractile mechanism involves associations between the myosin heads and actin filaments in non-muscle cells (23). Electron microscopy studies have established that myosin and actin filament co-localization is not uniform throughout the cell. Experiments with the isolated brush border have demonstrated that conformation of myosin is determined by the functional state of the tissue at the time of fixation (23). When myosin is phosphorylated, it is released from the actin cytoskeleton and is free to assemble into bipolar filaments (23). Following contraction, myosin is dephosphorylated by a phosphatase that restores myosin to its largely unpolymerized form and it becomes again attached to the actin cytoskeleton (26).

MLCK is the primary regulator of various forms of eukaryotic motility. MLCK serves a role in numerous functions of non-muscle cells, including cytoskeletal clustering, platelet shape alterations, transepithelial permeability and cytoskeletal arrangements, stress fiber formation, cell spreading and migration, cytokinesis, secretion and neurite growth cone advancement, which affect ion exchange or ion currents at the plasma membrane (27-29). In synapses, MLCK and its downstream substrate myosin, regulate different stages of neurotransmitter release (30), including refilling of readily releasable pools following tetanus stimulation, vesicle mobility (31,32), supply of fast-releasing vesicles (33) and vesicle mobilization (34). MLCK is required for activity-dependent functions of myosin in hippocampal neurons (35). Certain cellular effects of MLCK result from its phosphorylation of MLC (36).

The molecular mechanism underlying neuronal damage in hyperglycemia has not yet been fully elucidated. In the authors' previous study, a proteomic approach, reverse transcription PCR, western blot and immunohistochemistry assays were used to identify differential proteins expressed in the brain of rats with type 1 diabetes mellitus in vivo, and it was determined that MLCK is upregulated in rats with type $1 \mathrm{DM}$ (7). The authors also previously demonstrated that the degree of cognitive dysfunction in diabetic rats is aggravated in weeks 6-12 following induction of type 1 DM (37). The ultrastructure of hippocampal neurons is markedly altered and rate of cell apoptosis increases (37). The number of organelles decreases and nuclei exhibit increased intensity of staining (37). In week 12 following induction of the model of type 1 DM, microscopic observation revealed that the number of hippocampal neurons decreases markedly and the morphology of hippocampal neurons is abnormal (37). The authors previous study also demonstrated that the structure of the cerebral neurons distinctly degenerates and the expression of MLCK is upregulated in diabetic rats, and MLCK may serve a role in neuronal plasticity (38). The present study analyzed the ultrastructure of primary cultured hippocampal neurons treated with high glucose ( $45 \mathrm{mmol} / \mathrm{l})$ by TEM and it was demonstrated that the nuclear membranes had shrunk, the morphology of the nucleus was irregular, and the chromatins were heterogeneously distributed, granular and aggregated into blocks. Cytoplasm fragmentation, distinctly edematous endoplasmic reticulum and markedly enlarged mitochondria appeared, however the MLCK inhibitor ML-7 was able to reverse these alterations. Exposure to high-glucose conditions resulted in F-actin depolymerization and rearrangement, however ML-7 reversed this effect. Protein expression of MLCK and p-MLC was also determined in differentially treated hippocampal neurons and it was demonstrated that MLCK and p-MLC were upregulated in the high-glucose environment. Following addition of ML-7, the expression of MLCK and p-MLC was downregulated.

In conclusion, the present study demonstrated that high glucose upregulated the expression of MLCK to promote F-actin depolymerization, which, in turn, induced microfilament cytoskeleton rearrangement in hippocampal neurons. It is necessary to elucidate the pathogenesis of diabetic cognitive dysfunction in order to design novel modalities for clinical treatment, and the present study contributed to these efforts.

\section{Acknowledgements}

Not applicable.

\section{Funding}

The present study was supported by grants from the National Natural Science Funding (grant nos. 81560720 and 81560138).

\section{Availability of data and materials}

All data generated or analyzed during the present study are included in this published article.

\section{Authors' contributions}

All authors take public responsibility for the integrity of the data and the accuracy of analysis in the study. WP and XL made substantial contributions to the concept and design of the present study. LZ and CL made substantial contributions to the acquisition, analysis and interpretation of data. LZ drafted the manuscript. LZ conducted statistical analysis. GD bred the mice, and prepared and cultured hippocampal neuronal cells. MP identified the hippocampal neurons and carried out Western blot analysis. GL carried out immunofluorescence study and cell apoptosis analysis.

\section{Ethics approval and consent to participate}

The present study was approved by the Ethics Committee of Guizhou Medical University (approval no. 1702093).

\section{Consent for publication}

No applicable.

\section{Competing interests}

The authors declare that they have no competing interests. 


\section{References}

1. Duelli R, Maurer MH, Staudt R, Heiland S, Duembgen L and Kuschinsky W: Increased cerebral glucose utilization and decreased glucose transporter Glut1 during chronic hyperglycemia in rat brain. Brain Res 858: 338-347, 2000.

2. Malone JI, Hanna S, Saporta S, Mervis RF, Park CR, Chong L and Diamond DM: Hyperglycemia not hypoglycemia alters neuronal dendrites and impairs spatial memory. Pediatr Diabetes 9: 531-539, 2008.

3. Ahtiluoto S, Polvikoski T, Peltonen M, Solomon A, Tuomilehto J, Winblad B, Sulkava R and Kivipelto M: Diabetes, Alzheimer disease, and vascular dementia: A population-based neuropathologic study. Neurology 75: 1195-1202, 2010.

4. Summers WK: Alzheimer's disease, oxidative injury, and cytokines. J Alzheimers Dis 6: 651-657; discussion 673-681, 2004.

5. Pasquier F, Boulogne A, Leys D and Fontaine P: Diabetes mellitus and dementia. Diabetes Metab 32: 403-414, 2006.

6. Lee A, Fischer RS and Fowler VM: Stabilization and remodeling of the membrane skeleton during lens fiber cell differentiation and maturation. Dev Dyn 217: 257-270, 2000.

7. Li X, Pan W, Yang GZ, Di YN, Zhao F, Zhu LY and Jiang ZH Proteome analysis of differential protein expression in brain of rats with type 1 diabetes mellitus. Exp Clin Endocrinol Diabetes 119: 265-270, 2011

8. Zhang Y, Tian Q, Zhang Q, Zhou X, Liu S and Wang JZ: Hyperphosphorylation of microtubule-associated tau protein plays dual role in neurodegeneration and neuroprotection. Pathophysiology 16: 311-316, 2009.

9. Kruger L and Mandelkow EM: Tau neurotoxicity and rescue in animal models of human tauopathies. Curr Opin Neurobiol 36: $52-58,2016$.

10. SantacruzK,Lewis J, Spires T,Paulson J, Kotilinek L, Ingelsson M, Guimaraes A, DeTure M, Ramsden M, McGowan E, et al: Tau suppression in a neurodegenerative mouse model improves memory function. Science 309: 476-481, 2005.

11. McLean WG, Pekiner C, Cullum NA and Casson IF: Posttranslational modifications of nerve cytoskeletal proteins in experimental diabetes. Mol Neurobiol 6: 225-237, 1992.

12. Zhang J and Dong XP: Dysfunction of microtubule-associated proteins of MAP2/tau family in prion disease. Prion 6: 334-338, 2012.

13. Chen J, Liang L, Zhan L, Zhou Y, Zheng L, Sun X, Gong J, Sui H, Jiang $R$, Zhang $F$ and Zhang L: ZiBuPiYin recipe protects db/db mice from diabetes-associated cognitive decline through improving multiple pathological changes. PLoS One 9: e91680, 2014.

14. Yuan Q, Zhan L, Zhou QY, Zhang LL, Chen XM, Hu XM and Yuan XC: SIRT2 regulates microtubule stabilization in diabetic cardiomyopathy. Eur J Pharmacol 764: 554-561, 2015.

15. Stull JT, Kamm KE and Vandenboom R: Myosin light chain kinase and the role of myosin light chain phosphorylation in skeletal muscle. Arch Biochem Biophys 510: 120-128, 2011

16. Gao N, Tsai MH, Chang AN, He W, Chen CP, Zhu M, Kamm KE and Stull JT: Physiological vs. pharmacological signalling to myosin phosphorylation in airway smooth muscle. J Physiol 595 6231-6247, 2017.

17. Khapchaev AY and Shirinsky VP: Myosin light chain kinase MYLK1: Anatomy, interactions, functions, and regulation. Biochemistry (Mosc) 81: 1676-1697, 2016.

18. Martinsen A, Dessy C and Morel N: Regulation of calcium channels in smooth muscle: New insights into the role of myosin light chain kinase. Channels (Austin) 8: 402-413, 2014.

19. Wilcox KS, Buchhalter J and Dichter MA: Properties of inhibitory and excitatory synapses between hippocampal neurons in very low density cultures. Synapse 18: 128-151, 1994.

20. Gaspar JM, Castilho A, Baptista FI, Liberal J and Ambrosio AF: Long-term exposure to high glucose induces changes in the content and distribution of some exocytotic proteins in cultured hippocampal neurons. Neuroscience 171: 981-992, 2010.
21. Pardal R and Lopez Barneo J: Mature neurons modulate neurogenesis through chemical signals acting on neural stem cells. Dev Growth Differ 58: 456-462, 2016.

22. Yarden-Rabinowitz Y and Yarom Y: In vivo analysis of synaptic activity in cerebellar nuclei neurons unravels the efficacy of excitatory inputs. J Physiol 595: 5945-5963, 2017.

23. Fifkova E: Actin in the nervous system. Brain Res 356: 187-215, 1985.

24. He Q and Roblodowski C: Functional analysis of actin-binding proteins in the central nervous system of drosophila. Methods Mol Biol 1365: 349-355, 2016.

25. Liu L, Luo M, Yang B, Wu X, Zhu W, Guan Y, Cai W, Troidl K, Schaper W and Schaper J: Actin-binding rho activating protein is expressed in the central nervous system of normal adult rats. Neural Regen Res 7: 965-970, 2012.

26. Broschat KO, Stidwill RP and Burgess DR: Phosphorylation controls brush border motility by regulating myosin structure and association with the cytoskeleton. Cell 35: 561-571, 1983.

27. Bresnick AR: Molecular mechanisms of nonmuscle myosin-II regulation. Curr Opin Cell Biol 11: 26-33, 1999.

28. Aromolaran AS, Albert AP and Large WA: Evidence for myosin light chain kinase mediating noradrenaline-evoked cation current in rabbit portal vein myocytes. J Physiol 3: 853-863, 2000.

29. Yang X, Wang JG, Ma DB, Ma XF, Zhu GJ, Zhou H, Yu CJ, Qian XY and Gao X: Myosin light chain kinase regulates hearing in mice by influencing the F-actin cytoskeleton of outer hair cells and cochleae. Int J Mol Med 33: 905-912, 2014.

30. Mochida S, Kobayashi H, Matsuda Y, Yuda Y, Muramoto K and Nonomura Y: Myosin II is involved in transmitter release at synapses formed between rat sympathetic neurons in culture. Neuron 13: 1131-1142, 1994.

31. Lee JS, Kim MH, Ho WK and Lee SH: Presynaptic release probability and readily releasable pool size are regulated by two independent mechanisms during posttetanic potentiation at the calyx of Held synapse. J Neurosci 28: 7945-7953, 2008.

32. Peng A, Rotman Z, Deng PY and Klyachko VA: Differential motion dynamics of synaptic vesicles undergoing spontaneous and activity-evoked endocytosis. Neuron 73: 1108-1115, 2012.

33. Garcia-Morales V, Montero F, Gonzalez-Forero D, Rodriguez-Bey G, Gomez-Perez L, Medialdea-Wandossell MJ, Dominguez-Vias G, Garcia-Verdugo JM and Moreno-Lopez B: Membrane-derived phospholipids control synaptic neurotransmission and plasticity. PLoS Biol 13: e1002153, 2015.

34. Seabrooke S and Stewart BA: Synaptic transmission and plasticity are modulated by nonmuscle myosin ii at the neuromuscular junction of drosophila. J Neurophysiol 105: 1966-1976, 2011.

35. Li L, Wu X, Yue HY, Zhu YC and Xu J: Myosin light chain kinase facilitates endocytosis of synaptic vesicles at hippocampal boutons. J Neurochem 138: 60-73, 2016.

36. Somlyo AP and Somlyo AV: Ca2+ sensitivity of smooth muscle and nonmuscle myosin II: Modulated by G proteins, kinases, and myosin phosphatase. Physiol Rev 83: 1325-1358, 2003.

37. Wang Feng, He Yu, Liu Mi, Chang Xiao, Ren Zhijing, Pan Wei and Li Xing: The establishment of the diabetic rat model with cognitive dysfunction and the observation of cognitive dysfunction in the diabetic rats. Chongqing Medicine 16: 2234-2236, 2015.

38. He Y, Wang F, Chen S, Liu M, Pan W and Li X: The protective effect of radix polygoni multiflori on diabetic encephalopathy via regulating myosin light chain kinase expression. J Diabetes Res 2015: 484721, 2015.

This work is licensed under a Creative Common Attribution-NonCommercial-NoDerivatives 4.0 International (CC BY-NC-ND 4.0) License. 